\title{
Fidelity of HIV programme implementation by community health workers in rural Mopani district, South Africa: a community
}

\section{survey}

Nireshni Naidoo ${ }^{1,2}$, Jean P. Railton', Sellina N. Khosa', Nthabiseng Matlakala', Gert Marincowitz³, James A. Mclntyre ${ }^{1,4}$, Helen E. Struthers ${ }^{1,5}$, Jude Igumbor $^{2}$ and Remco P. H. Peters ${ }^{1,2,6^{*}}$ (i)

\begin{abstract}
Background: South Africa has implemented a community health programme delivered by community health workers (CHWs) to strengthen primary healthcare services. Provision of community Human Immunodeficiency Virus (HIV) services constitutes an important component of this programme. To support effectiveness, we assessed fidelity of HIV programme implementation by CHWs from the community's perspective in a rural South African setting.

Methods: A cross-sectional study was conducted targeting 900 randomly selected households in twelve wards of two sub-districts (Greater Giyani and Greater Letaba) of Mopani District (Limpopo Province, South Africa). Questionnaires were administered to the traditionally most appropriate adult member of the household. Included were questions related to the four standard components to measure implementation fidelity against local guidelines: coverage, frequency, duration and content of HIV programme implementation.

Results: Participants were enrolled at 534 households; in most other cases there was nobody or no adult member at home $(n=291)$. Reported coverage of 55\% (141/253) and a frequency of $47 \%(66 / 140)$ were higher in Greater Giyani as compared to Greater Letaba (44\%; 122/278 and 29\%; 33/112, respectively, $p=0.007$ for both comparisons). Coverage was not associated with the distance from the participant's household to the facility $(p=0.93)$. Duration of programme delivery was reported to be high, where all CHW visits (253/253; 100\%) were conducted within the last 6 months and the content delivered was adequate (242/253; 96\%). Individuals reporting a CHW visit were more likely to know their HIV status than those not visited ( $\mathrm{OR}=2.0 ; 95 \% \mathrm{Cl} 1.06-3.8 ; p=0.032)$. Among those visited by the CHW discussion of HIV was associated with knowing the HIV status $(\mathrm{OR}=2.2 ; 95 \% \mathrm{Cl} 1.02-4.6 ; p=0.044)$; in particular for women $(\mathrm{OR}=2.9$; $95 \% \mathrm{Cl} 1.5-5.4 ; p=0.001)$.

Conclusions: This study demonstrates promising HIV programme implementation fidelity by CHWs in rural South Africa. Programme coverage and frequency should be improved whilst maintaining the good levels of duration and content. Resource investment, strengthening of operational structure, and research to identify other facilitators of programme implementation are warranted to improve programme effectiveness and impact.
\end{abstract}

Keywords: Community health workers, HIV, Implementation, Community, Public health

\footnotetext{
* Correspondence: peters@anovahealth.co.za; rph.peters@gmail.com

${ }^{1}$ Anova Health Institute, PostNet Suite 242, Private Bag X30500, 2041

Houghton, Johannesburg, South Africa

${ }^{2}$ Division of Epidemiology and Biostatistics, School of Public Health, Faculty

of Health Sciences, University of the Witwatersrand, Johannesburg, South

Africa

Full list of author information is available at the end of the article
}

(c) The Author(s). 2018 Open Access This article is distributed under the terms of the Creative Commons Attribution 4.0 International License (http://creativecommons.org/licenses/by/4.0/), which permits unrestricted use, distribution, and reproduction in any medium, provided you give appropriate credit to the original author(s) and the source, provide a link to the Creative Commons license, and indicate if changes were made. The Creative Commons Public Domain Dedication waiver (http://creativecommons.org/publicdomain/zero/1.0/) applies to the data made available in this article, unless otherwise stated. 


\section{Background}

Community health programmes have strong potential to strengthen primary health services in low- and middle income countries (LMICs) [1]. The value of these programmes has been demonstrated for maternal, child, and mental healthcare in various settings in LMICs. For example, studies from South Africa show that CHWs have been successful in improving maternal and child health outcomes [2], and providing a social support system [3]. Reviews of quantitative and qualitative studies show that CHWs can provide an important human resource capacity and have the potential to contribute to HIV services in sub-Saharan Africa $[4,5]$. There is growing evidence on successful scale up and integration of community-based programmes into national health systems [6-8]. However, despite its strong potential, only limited data are available with regard to provision of HIV services at community level $[9,10]$.

South Africa, the country with the largest HIV programme in the world, has implemented a community health programme since 2012 with the aim to improve access to and provision of high-quality primary healthcare (PHC) services [11]. South Africa's community health programme is delivered through ward-based outreach teams (WBOTs), each comprising a professional nurse that serves as team leader and up to five community health workers (CHWs) [11]. The local guidelines stipulate that the team of five CHWs should support a population of 7660 individuals in total [11]. The services provided by the CHWs include general health education, health status monitoring, and referral of individuals in need of care to the PHC facility [11].

There is a strong programmatic focus on vulnerable populations, including individuals with chronic illnesses; HIV or tuberculosis (TB); pregnant women; as well as screening for malnutrition and gastroenteritis; and checking immunisation, vitamin $\mathrm{A}$ and deworming status in children.

Provision of HIV services constitutes an important component in the community health programme. The CHWs contribute to HIV programme delivery through providing health education to prevent HIV infection, identifying individuals that need to test for HIV, referring HIV-infected individuals not yet in care to start antiretroviral therapy (ART), providing adherence support to those on ART, tracing and referral of HIV-infected individuals that have been lost to the ART programme, and identifying individuals who have clinically failed on ART and require further assessment [11]. Visits to HIV-infected individuals should happen on a monthly basis while assessment of HIV risk and potential referral for testing should happen at least annually [11].

Regular assessment and monitoring of implementation performance of any health programme is essential to obtain maximum impact of its implementation and to ensure that the programme meets its intended goals [12]. The same holds true for the HIV programme as provided by CHWs. However, to the best of our knowledge there is no record of a comprehensive assessment of implementation of this programme in South Africa [13]. Studies from other countries, not addressing HIV, show that lack of such information could negatively affect programme effectiveness [2-4]. Knowledge of how and why a community health programme works, or does not work, in terms of implementation is required to optimise programme implementation before successful scale-up [1, 6, 14].

An important method to assess implementation of health programmes is to measure fidelity to the intended programme specifics. Fidelity to programme implementation is generally measured on four aspects: 'coverage' (services provided to everyone that is supposed to receive these services), 'frequency' (services provided by the prescribed frequency), 'duration' (service delivery that is uninterrupted) and 'content' (the correct services provided) [15]. In our context, this means measurement of HIV programme implementation by CHWs of these four components against the programme specification in the local government guidelines [11]. In this study we aim to measure fidelity to HIV programme implementation as provided by CHWs from the community's perspective in rural Mopani District, South Africa. We conducted a community survey to obtain insight into the current status of programme implementation and to identify areas that warrant strengthening to improve effectiveness of HIV service delivery by CHWs.

\section{Methods}

\section{Study setting and design}

The community health programme was initiated in Mopani district in 2013 and, as of mid-2016, is provided by 149 WBOTs that cover 123 of the 125 wards (98\%) and $75 \%$ of registered households. [16]. All CHWs are female. A cross-sectional study was conducted from May to July 2016 in two sub-districts (Greater Giyani and Greater Letaba) of the Mopani district, Limpopo province, South Africa. Mopani district is one of the most rural districts in the country and is considered to be one of the main infrastructure intervention areas for economic transformation in South Africa [17]. There are 83,225 and 54,228 households in Greater Giyani and Greater Letaba sub-district supported by 37 and 24 WBOTs, respectively. The WBOTs in these sub-districts have operational differences such as the number of CHWs deployed, workload, and team performance.

We purposively selected the communities living in twelve wards, six per sub-district. These wards were selected as each was the single ward draining to a PHC 
facility (some facilities have multiple wards draining into them) and the catchment population was of similar size $(+/-45,000$ individuals). Each of the twelve wards is supported by a single WBOT that has divided the ward into sections. Households in each section are supported by an individual CHW. For this study we randomly selected three of the sections in each ward (36 in total). We then randomly selected 25 households in each section by manually pinning households in Google ${ }^{\mathrm{mx}}$ Earth Pro. The Global Positioning System (GPS) coordinates of each selected household was recorded and given to the study team. We used coverage, i.e. report of a CHW household visit by the participant, as the main measure of outcome. Based on an estimated coverage of $80 \%$, using a confidence level of $95 \%$, and power of $80 \%$, we calculated a sample size of 734 individuals as adequate. Assuming a response rate of $80 \%$, a sample size of 900 was used. Ethical approval was obtained from the Human Research Ethics Committee at the University of Witwatersrand, Johannesburg, South Africa (Reference number: M1611111) as well as the Limpopo Provincial Health Research Committee.

\section{Study procedures}

The study team visited selected households using maps and GPS tracker to find the correct location. Individuals found at home were approached to participate and screened for eligibility. Individuals had to be adult (> 18 years) to participate; in the case that there were multiple people at home, the most senior individual, as culturally appropriate, was interviewed. Following written informed consent, a questionnaire was administered that included questions about the individual's demographic details, interactions with and perceptions of their $\mathrm{CHW}$ as well as the services received from them.

\section{Measurement of implementation fidelity}

Implementation fidelity was assessed by measuring content, coverage, frequency, and duration in relation to the provincial guidelines that are currently in place [11]. Coverage, 'reach', was measured by the proportion of households that reported CHW visits ever. Frequency was assessed by determining the proportion of households that received $\mathrm{CHW}$ visits according to the required schedule (at least once a month in case of vulnerable household). Duration of implementation refers to the need for ongoing service delivery, i.e. no major programme interruptions; we defined duration as high in case of CHW visit $<6$ months ago or low for $\mathrm{CHW}$ visit $>6$ months ago. Finally, content was measured by the proportion of individuals reporting HIV services delivered by CHWs that were deemed by the researchers in line with the guidelines, including HIV health education and referrals [11].

\section{Statistical analysis}

Data were collected using structured interview guides and double-captured into a database (EpiInfo $\left.{ }^{\mathrm{Tm}}\right)$. Validity checks were done to ensure completeness and to identify consistency and range errors. Socio-demographic characteristics of the households are described using proportions with confidence intervals and means with standard deviation. We determined factors associated with a $\mathrm{CHW}$ visit using univariate logistic regression. Variables with a $p$ value $\leq 0.1$ on univariate logistic regression were included in the multivariate logistic regression model as well as sub-district as expected confounding variable. We included social grant in our analysis as provided by the South African government to various groups (pensioners, disabled individuals, and child support).

\section{Results}

\section{Description of the study population}

The study team managed to visit $99 \%$ of the households (892/900) randomly selected for this study; in the other 8 cases there was no longer a house at the location identified on the map. Participants were enrolled at 534/892 $(60 \%)$ households; there was no difference in enrolment between Greater Giyani (49.6\%) and Greater Letaba $(50.3 \%)$ sub-districts $(p=0.79)$. At the non-included households (358), there was nobody at home in 275 (31\%) cases or only a child at home (16; $1.8 \%)$. Participation was declined at 47 households $(5.3 \%)$ for other reasons (Fig. 1). The majority of participants was female (410/534; 77\%) and median age was 46 years (range 1896) (Table 1). Social grants were the main source of income reported by participants in both sub-districts, $52 \%$ $(137 / 265)$ and $43 \%(117 / 269)$ in Greater Giyani and Greater Letaba respectively $(p=0.028)$.

\section{Implementation fidelity: measurement of coverage, frequency, and duration}

In terms of coverage, participants from 253/534 (47\%) households reported to have ever been visited by a CHW (Table 2), of which 100\% (253/253) were visited in the last 6 months. A CHW visit was more often reported in Greater Giyani (55\%) than in Greater Letaba (45\%) sub-district $(\mathrm{OR}=1.6 ; 95 \% \mathrm{CI} 1.14-2.27 ; p=0.007)$. This is possibly due to the fact that there are 54 and 36 CHWs deployed on Greater Giyani and Greater Letaba, respectively, and that Greater Giyani has better organisational structures. Individuals 60 years and older were more likely to be visited by a CHW as compared to individuals 18 to 34 years of age $(\mathrm{OR}=1.7 ; 95 \%$ CI 1.08 2.77; $p=0.022$. There was no association of reporting a $\mathrm{CHW}$ visit with distance between household and nearest facility $(1.4 \mathrm{~km}$ for visited vs. $1.6 \mathrm{~km}$ for not visited; $p=$ $0.93)$. We plotted households on the map by status of CHW visit reported, and did not observe any obvious 


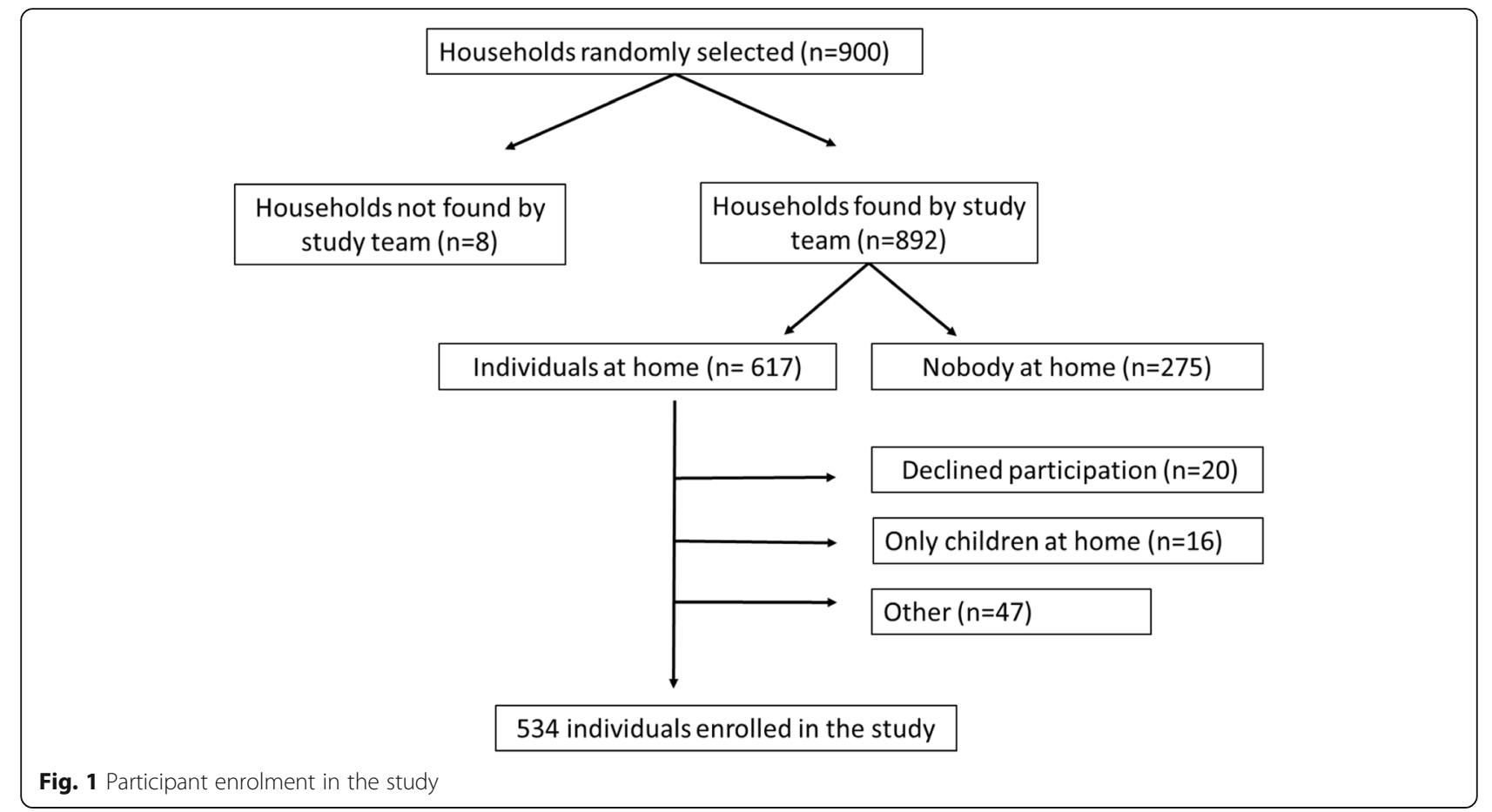

patterns or clusters that could indicate other geographic factors. Most participants who reported a CHW visit were happy with such visit $(192 / 253 ; 76 \%)$ whereas a large proportion of participants not visited by a $\mathrm{CHW}$ would have liked such a visit $(206 / 278 ; 74 \%)$. Participants who had been visited by a CHW were more likely to know their HIV status $(238 / 253 ; 94 \%)$ compared to those not visited $(245 / 278$; 88\%) $(\mathrm{OR}=2.0$; 95\% CI $1.06-$
3.8; $p=0.032$ ); there was no statistically significant difference between whether or not a household was visited and gender $(\mathrm{OR}=1.4 ; 95 \% \mathrm{CI} 0.92-2.08 ; p=0.118)$.

In terms of frequency, 39\% (99/253) of participants overall reported that the $\mathrm{CHW}$ visit had occurred in the previous month as per provincial guideline. However, frequency of a CHW visit in the previous month was $47 \%(66 / 140)$ in Greater Giyani and only 29\% (33/112)

Table 1 Characteristics of the study population $(n=534)$

\begin{tabular}{|c|c|c|c|c|}
\hline Variable & & Greater Giyani $(N=265)$ & Greater Letaba $(N=269)$ & Total \\
\hline \multirow[t]{2}{*}{ Gender } & Male & $61(23 \%)$ & $63(23 \%)$ & 124 \\
\hline & Female & $204(77 \%)$ & $206(77 \%)$ & 410 \\
\hline \multirow[t]{4}{*}{ Source of income } & Formal employment & $63(24 \%)$ & $92(34 \%)$ & 155 \\
\hline & Informal work & $56(21 \%)$ & $58(22 \%)$ & 114 \\
\hline & Social grants & $137(52 \%)$ & $117(43 \%)$ & 254 \\
\hline & Other & $9(3 \%)$ & $2(1 \%)$ & 11 \\
\hline \multirow[t]{4}{*}{ Primary member in the household that takes of the children } & Mother & 77 (29\%) & 79 (29\%) & 156 \\
\hline & Father & $76(29 \%)$ & $74(28 \%)$ & 150 \\
\hline & Grandmother & $50(19 \%)$ & $60(22 \%)$ & 110 \\
\hline & Other & $62(23 \%)$ & $56(21 \%)$ & 118 \\
\hline \multirow[t]{3}{*}{ Age of participants (years) } & $18-34$ & $88(33 \%)$ & $90(34 \%)$ & 178 \\
\hline & $35-59$ & $90(34 \%)$ & $119(44 \%)$ & 209 \\
\hline & $\geq 60$ & $87(33 \%)$ & $60(22 \%)$ & 147 \\
\hline aMedian number of individuals living in household & & $5(0-10)$ & $6(0-10)$ & 5.5 \\
\hline aMedian distance from participant's house to the clinic (km) & & $1.4(0-13)$ & $1.6(0-11)$ & 1.5 \\
\hline
\end{tabular}

\footnotetext{
${ }^{a}$ Numbers presented in median (range)
} 
Table 2 Univariate and multivariate analysis of factors associated with participant reporting CHW visit

\begin{tabular}{|c|c|c|c|c|c|c|c|}
\hline Variable & & Visited by CHW & Not visited by CHW & Unadjusted OR (95\% Cl) & $P$-value & Adjusted OR (95\% Cl) & $P$-value \\
\hline Number & & $253(48 \%)$ & $278(52 \%)$ & & & & \\
\hline \multirow[t]{2}{*}{ Sub-district } & Giyani & $141(55 \%)$ & $122(44 \%)$ & $1.7(1.14-2.27)$ & 0.007 & $1.6(1.14-2.27)$ & 0.007 \\
\hline & Letaba & $112(45 \%)$ & $156(56 \%)$ & Ref & Ref & Ref & Ref \\
\hline Distance to clinic (km) & & $1.4(0-13)$ & $1.6(0-11)$ & & 0.934 & - & - \\
\hline \multirow[t]{2}{*}{ Gender } & Male & $51(20 \%)$ & $72(26 \%)$ & Ref & Ref & -- & - \\
\hline & Female & $202(80 \%)$ & 206 (74\%) & $1.4(0.92-2.08)$ & 0.118 & - & - \\
\hline \multirow[t]{3}{*}{ Age-group (years) } & 18-34 years & 75 (30\%) & $100(36 \%)$ & Ref & Ref & Ref & Ref \\
\hline & $35-59$ years & 95 (37\%) & $114(41 \%)$ & $1.1(0.74-1.67)$ & 0.610 & $1.2(0.76-1.76)$ & 0.503 \\
\hline & $\geq 60$ years & $83(33 \%)$ & $64(23 \%)$ & $1.7(1.11-2.69)$ & 0.015 & $1.7(1.08-2.77)$ & 0.022 \\
\hline \multirow[t]{3}{*}{ Source of income } & Formal & $70(28 \%)$ & $83(30 \%)$ & Ref & Ref & - & - \\
\hline & Informal & $49(19 \%)$ & $65(23 \%)$ & $0.9(0.52-1.41)$ & 0.546 & - & - \\
\hline & Grants & $129(51 \%)$ & $124(45 \%)$ & $1.2(0.82-1.87)$ & 0.313 & - & - \\
\hline \multirow[t]{3}{*}{ Participant knowledge of HIV } & Yes & $229(91 \%)$ & $231(83 \%)$ & $2.1(1.08-3.90)$ & 0.029 & $2.0(1.06-3.8)$ & 0.032 \\
\hline & No & $15(6 \%)$ & $31(11 \%)$ & Ref & Ref & Ref & Ref - \\
\hline & Unknown & $9(4 \%)$ & $15(5 \%)$ & - & - & - & - \\
\hline
\end{tabular}

Abbreviations: $\mathrm{CHW}$ community health workers, OR odds ratio, $\mathrm{Cl}$ confidence interval

"Participants that refused to answer whether they had been visited by a CHW or not were omitted from the analysis ( $n=3$ ). Statistically significant factors in bold

in Greater Letaba district $(p=0.007)$. There was no association of any demographic factors with frequency. In terms of duration of HIV programme implementation, all participants who had been visited by a CHW (253/ 253; 100\%) reported high duration in both sub-districts (last visit $<6$ months ago).

\section{Implementation fidelity: measurement of content}

Ninety-six percent $(242 / 253)$ of participants visited by a $\mathrm{CHW}$ had discussed any type of health issue during the last visit: $67 \%(170 / 253)$ reported having discussed HIV with the CHW, 66\% (167/253) TB, 74\% (187/253) chronic illnesses other than HIV, 11\% (28/253) pregnancy, and 55\% (139/253) other health-related issues. Most participants, $89 \%$ (225/253), were satisfied with the content of services rendered by the CHW.

Participants were twice as likely to know their HIV status if HIV had been discussed with them by the CHW compared to those with whom HIV was not discussed $(\mathrm{OR}=2.2 ; 95 \%$ CI 1.02-4.60; $p=0.044)$. Females were twice as likely to know their HIV status as compared to men $(\mathrm{OR}=2.9 ; 95 \%$ CI $1.5-5.4 ; p=0.001)$. There was no association for women of knowing their HIV status and having discussed HIV with CHWs (OR $=1.5$; $95 \% \mathrm{CI}$ $0.6-3.5 ; p=0.401$ ); for men there was a positive trend $(\mathrm{OR}=4.2 ; 95 \%$ CI $0.9-19.1 ; p=0.066)$. In terms of referring participants for further screening or treatment, $20 \%$ $(51 / 253)$ of participants reported that a CHW had referred the participant or another household member to the nearest facility during the last visit for HIV testing (23/51; 45\%), TB screening $(7 / 51 ; 14 \%)$ or an assessment of other chronic illnesses $(20 / 51 ; 39 \%)$ and pregnancy testing $(1 / 51 ; 1 \%)$. Of these referrals, $88 \%(45 / 51)$ reported to have visited the clinic for healthcare.

\section{Discussion}

This is the first study to measure fidelity of implementation of the HIV programme as provided by CHWs in rural South Africa. We are not aware of any other HIV programme with fidelity data from similar settings to provide a comparison to our observations. However, when compared to other South African and global studies measuring $\mathrm{CHW}$ chronic disease services other than HIV, we observed similar findings with regard to duration and content, and similarly found that coverage and frequency should be improved [18-20].

In terms of coverage and frequency, we measured a $47 \%$ coverage of households from which the participant reported a CHW visit at any point in time, despite the policy that all households ought to be visited on an annual basis to assess vulnerability status. This is lower than reported by studies of chronic diseases that showed coverage in the range of $57 \%$ to $74 \%[19,20]$, but good compared to a systematic review of 38 studies that reported $18 \%$ coverage for community health programmes overall [18]. In addition, frequency of visits was relatively low at $39 \%$, but this was similar to other studies [18]. Increasing coverage and frequency of $\mathrm{CHW}$ visits could directly impact on the HIV programme as participants who had been visited by CHWs were more likely to have discussed HIV and know their HIV status than those that had not been seen by a CHW. Various factors may have impacted on coverage and frequency in our area. First, operational factors may play a role: coverage was 
higher in Greater Giyani sub-district where in our opinion the programme stronger managed and where there are on average more CHWs employed in each community than in Greater Letaba (resulting in lower number of households covered by each $\mathrm{CHW}$ ). Second, an important factor to consider is the capacity of an individual CHW to visit all allocated households in the section within the set timeframe. It is possible that in our rural area, the target number of 270 households, which in practice becomes $250-400$ households, is too large to cover within the specific programme deliverables. Reduction of the number of households allocated to each $\mathrm{CHW}$ by increasing the number of CHWs per ward should be considered to improve coverage [11]. Third, geographic factors such as houses located in rocky ad mountainous areas may impact on accessibility of houses to CHWs. In addition, challenging terrain would likely take the $\mathrm{CHW}$ more time to reach a household, limiting the number of households that can be visited during a day. Although an effect cannot be ruled out, we did not observe any obvious patterns or clusters when looking at households reporting visits compared to those not visited. Distance between the household and nearest PHC facility was not associated with the report of a CHW visit as most houses were within walking distance to the facility. Despite the need to improve coverage and frequency, we observed high appreciation of CHW visits by the community; most participants were happy with the CHW visits and services provided, whereas most of those that had not been visited by the CHW would really appreciate a visit. We did not observe a difference between male and female respondents with regards to interest in and appreciation of $\mathrm{CHW}$ visits. This contradicts findings from another report from Pakistan that suggests that men are unwilling to interact with the female CHWs [21].

In terms of duration and content, we measured high duration (100\%) of programme implementation among those visited by the CHWs as compared 39\% reported the aforementioned systematic review of 38 studies [18]. This suggests that services are relatively continuous and uninterrupted once there is a structural relationship between household members and CHWs. In addition, the content of service provision for HIV was 67\%) and no major gaps were identified. This is good when compared to a systematic review of 38 studies that reported content provision of chronic diseases services in the range of $40 \%$ to $66 \%[18,19]$. This indirectly shows the impact of the intensive CHW training programme (phase 1 and phase 2 of the national curriculum) that was conducted in Mopani District [11]. Participants with whom HIV was discussed were more likely to know their HIV status suggesting a direct link between CHW activities and HIV programme performance. We did not distinguish between HIV-infected and HIV-uninfected participants.
However, HIV should be discussed with all household members.

This study shows that despite limited resources, there is a reasonably good implementation of HIV programme activities by CHWs in our rural South African district. Although coverage and frequency should be improved, there is good content and duration of service provision to those that are reached by the CHWs. This supports the high promise of CHW implementation of the HIV programme on a structural basis. Resource investment in the $\mathrm{CHW}$ programme, through increasing the number of CHWs and strengthening operational structures, may have to be expanded to achieve the programme's full potential and effectiveness, the sub-district with the lower resources also scored lower in implementation fidelity. These findings are consistent with other studies showing that adequate staffing, resource provision, and a human resource management approach are imperative for successful implementation of community-based programmes [22, 23]. In light of this, the use of human resource strategies such as clarification of roles, job satisfaction, and adequate remuneration should be considered to improve the South African CBHP. Although operational determinants may play a role, other factors that contribute to the programme success or provide a barrier to successful coverage and frequency need to be determined within a local context, in order to further improve implementation fidelity. Moreover, alternate strategies should be considered to reach the large proportion of households where we found no one at home, such as worksite wellness and local media initiatives [24].

This study has several limitations. Firstly, several types of bias may have occurred including recall and desirability bias. Selection bias may have also occurred as there was nobody at home at a substantial proportion of households. Possibly, these represent higher socio-economic group as they may be at work during the day. Although we recruited more women than men, there was no difference in their response. The response rate could have possibly been increased by visiting households multiple times instead of once. However, this was not feasible for operational reasons. Secondly, we have measured implementation fidelity from the community's (recipient) perspective. Triangulation of our findings with similar measurement from the CHW's perspective could have strengthened our findings, but resources did not permit this. We did not allocate a health vulnerability status to the households included in this study, which would have allowed for a more precise assessment of some components of implementation fidelity. This was due to the fact that household registration data, including vulnerability status, were not available through the CHWs at the time of study and could not be reliably collected as part of the questionnaire. For operational purpose, we collected data on CHW visits within 
the last 6 months as opposed to the recommended 12 months, which may have resulted in an underestimation of frequency. Finally, a time component was not included in the question where individuals were asked if they knew their HIV status.

\section{Conclusions}

In conclusion, this study shows that implementation fidelity of the HIV programme as provided by CHWs in a rural South Africa district is similar to other CHW programmes that report on chronic illnesses, other than HIV. There is room for improvement, in particular of coverage and resource investment is required to increase frequency of household visits. While this study informs policy makers on specific programme areas to improve this CBHP, further quantitative and qualitative research in similar contexts is required to further gauge barriers and facilitators to the fidelity of CHBP implementation.

\section{Abbreviations}

ART: Antiretroviral therapy; CHWs: Community health workers; GPS: Global Positioning System; HIV: Human Immunodeficiency Virus; LMICs: Low-and middle income countries; PHC: Primary healthcare; TB: Tuberculosis; WBOTs: Ward-based outreach teams

\section{Acknowledgements}

We thank all the participants who participated in this study. Our appreciation also goes to the staff of the Anova Health Institute for their invaluable contribution, efforts and support.

\section{Funding}

This study is made possible by the generous support of the American people through the US President's Emergency Plan for AIDS Relief (PEPFAR) through the United States Agency for International Development (USAID) under Cooperative Agreement number 674-A-12-00015 to the Anova Health Institute. The contents are the responsibility of Anova Health Institute and do not necessarily reflect the views of USAID or the United States Government. The funding body did not play a role in the design of the study, data collection, analysis, interpretation of data, and in writing the manuscript. This research project was supported by a postgraduate training scholarship from the Fogarty and NIAID, The UNC-Wits AIDS Implementation Science and Cohort Analyses Training Grant (Grant number: 5D43TW009774-02). The content is solely the responsibility of the authors and does not necessarily represent the official views of the Fogarty and NIAID.

\section{Availability of data and materials}

The datasets generated and analysed during this study are available from the corresponding author on reasonable request.

\section{Authors' contributions}

NN was responsible for planning the study, participated in its design and coordination, performed data cleaning, statistical analysis, and drafted the manuscript. JR participated in the design of the study and helped to draft the manuscript. SNK coordinated and carried out the cross-sectional study. NM coordinated and carried out the cross-sectional study. GM helped to draft the manuscript. JAM helped to draft the manuscript. HES helped to draft the manuscript. J helped to draft the manuscript. RPHP was responsible for planning of the study, participated in its design and coordination, and helped to draft the manuscript. All authors read and approved the final manuscript.

\section{Ethics approval and consent to participate}

Written informed consent was obtained from all study participants. Ethical approval was obtained from the Human Research Ethics Committee at the University of Witwatersrand, Johannesburg, South Africa (Reference number: M1611111) as well as the Limpopo Provincial Health Research Committee.
Consent for publication

Not applicable.

\section{Competing interests}

The authors declare that they have no competing interests.

\section{Publisher's Note}

Springer Nature remains neutral with regard to jurisdictional claims in published maps and institutional affiliations.

\section{Author details}

${ }^{1}$ Anova Health Institute, PostNet Suite 242, Private Bag X30500, 2041 Houghton, Johannesburg, South Africa. ${ }^{2}$ Division of Epidemiology and Biostatistics, School of Public Health, Faculty of Health Sciences, University of the Witwatersrand, Johannesburg, South Africa. ${ }^{3}$ Mopani District Specialist Team, Department of Health, Giyani, Limpopo Province, South Africa. ${ }^{4}$ School of Public Health \& Family Medicine, University of Cape Town, Cape Town, South Africa. ${ }^{5}$ Division of Infectious Diseases \& HIV Medicine, Department of Medicine, University of Cape Town, Cape Town, South Africa. ${ }^{6}$ Department of Medical Microbiology, School of Public Health and Primary Care (CAPHRI), Maastricht University Medical Centre (MUMC+), Maastricht, The Netherlands.

Received: 5 May 2018 Accepted: 1 August 2018

Published online: 06 September 2018

References

1. Horwood CM, Youngleson EM, Stern AF, PM B. Using adapted qualityimprovement approaches to strengthen community-based health systems and improve care in high HIV-burden sub-Saharan African countries. Report No: AIDS. 2015;29(Suppl 2):S155-64.

2. le Roux K, le Roux I, Mbewu N, Davis E. The role of community health Workers in the re-Engineering of primary health Care in Rural Eastern Cape. South African family practice : official journal of the South African Academy of Family Practice/Primary Care. 2015;57(2):116-20.

3. Moshabela M, Sips I, Barten F. Needs assessment for home-based care and the strengthening of social support networks: the role of community care workers in rural South Africa. Glob Health Action. 2015;8:29265.

4. Mwai GW, Mburu G, Torpey K, Frost P, Ford N, Seeley J. Role and outcomes of community health workers in HIV care in sub-Saharan Africa: a systematic review. J Int AIDS Soc. 2013;16:18586.

5. Naidoo N, Zuma N, Khosa S, Marincowitz G, Railton J, Matlakala N, et al. Qualitative assessment of facilitators and barriers to HIV Programme implementation by community health Workers in Mopani District, South Africa. Submitted.

6. Lui A, Sullivan $S$, Khan M, Sachs $S$, Singh P. Community health workers in global health: scale and scalability. Mt Sinai J Med. 2011. Report No.: 78: 419-435.

7. McCord GC, Liu A, Singh P. Deployment of community health workers across rural sub-Saharan Africa: financial considerations and operational assumptions. Bulletin of the World Health Organization. 2013;91(4):244-53b.

8. Pallas SW, Minhas D, Perez-Escamilla R, Taylor L, Curry L, Bradley EH. Community health workers in low- and middle-income countries: what do we know about scaling up and sustainability? Am J Public Health. 2013; 103(7):e74-82.

9. Johnson CD, Noyes J, Haines A, Thomas K, Stockport C, Ribas AN, et al. Learning from the Brazillian community health worker model in north whales. Report no. Glob Health. 2013;9:25.

10. Wajid A, White F, MS K. Community Health Workers and Healthcare Delivery: Evaluation of a Women's Reproductive Health Care Project in a Developing Country. PLoS ONE. 2013;8(9):e75476.

11. National Department of Health. Provincial Guidelines for the Implementation of the three streams of PHC Re-engineering. Pretoria: National Department of Health; 2011.

12. Dusenbury $L$, Brannigan $R$, Falco $M$, Hansen WB. A review of research on fidelity of implementation: implications for drug abuse prevention in school settings. Health Educ Res. 2003;18(2):237-56

13. Walt G. Commentary: lay health workers in primary and community health care. Int J Epidemiol. 2005;34(6):1251. discussion 2

14. Lehmann USD Community health workers: what do we know about them? The state of the evidence on programmes, activities costs and impact on 
health outcomes of using community health workers. Geneva: WHO Department of Health; 2007.

15. Carroll C, Patterson M, Wood S, Booth A, Rick J, Balain S. A conceptual framework for implementation fidelity. Implementation science: IS. 2007;2:40.

16. Marincowitz G. District Family Physician, Mopani District. Personal Communication ed 2016.

17. Deprtment of Rural Development and Land Reform. Priority Districts Analyses: High impact (catalytic) infrastructure intervention areas for economic transformation. 2013.

18. Aziz Z, Absetz P, Oldroyd J, Pronk NP, Oldenburg B. A systematic review of real-world diabetes prevention programs: learnings from the last 15 years. Implementation science : IS. 2015;10:172.

19. DePue JD, Rosen RK, Seiden A, Bereolos N, Chima ML, Goldstein MG, et al. Implementation of a culturally tailored diabetes intervention with community health workers in American Samoa. The Diabetes educator. 2013:39(6):761-71.

20. Namazzi G, Okuga M, Tetui M, Muhumuza Kananura R, Kakaire A, Namutamba S, et al. Working with community health workers to improve maternal and newborn health outcomes: implementation and scale-up lessons from eastern Uganda. Global health action. 2017;10(sup4):1345495.

21. Afsar HA, Qureshi AF, Younus M, Gulb A, Mahmood A. Factors affecting unsuccessful referral by the lady health Workers in Karachi, Pakistan. JPMA The Journal of the Pakistan Medical Association. 2003;53(11):521-8.

22. Austin-Evelyn K, Rabkin M, Macheka T, Mutiti A, Mwansa-Kambafwile J, Dlamini T, et al. Community health worker perspectives on a new primary health care initiative in the eastern cape of South Africa. PLoS One. 2017; 12(3):e0173863.

23. Raven J, Akweongo P, Baba A, Baine SO, Sall MG, Buzuzi S, et al. Using a human resource management approach to support community health workers: experiences from five African countries. Hum Resour Health. 2015;13(1):45.

24. Cosgrove S, Monroy M, Jenkins C, Castillo SR, Williams C, Parris E, et al. Community health workers as an integral strategy in the REACH U.S. program to eliminate health inequities. Health Promot Pract. 2014;15(6): 795-802.

Ready to submit your research? Choose BMC and benefit from:

- fast, convenient online submission

- thorough peer review by experienced researchers in your field

- rapid publication on acceptance

- support for research data, including large and complex data types

- gold Open Access which fosters wider collaboration and increased citations

- maximum visibility for your research: over $100 \mathrm{M}$ website views per year

At $\mathrm{BMC}$, research is always in progress.

Learn more biomedcentral.com/submissions 